\title{
Childhood Leukemias-Diagnosis, Treatment and New Agent Testing
}

a report by

\section{E Anders Kolb, MD}

Assistant Professor of Pediatrics, Division of Pediatric Hematology and Oncology,

The Children's Hospital at Montefiore and Albert Einstein College of Medicine

DOI: 10.17925/OHR.2006.00.01.59

The incremental increase in survival among children with acute lymphoblastic leukemia over the past 50 years is often cited as the great success story in clinical oncology. Leukemias are the most common malignancy of childhood, with an annual incidence of 40 cases per 1,000,000 people in the US, accounting for a quarter of all childhood malignancies. In the US, more that 3,000 children are diagnosed with leukemia each year, $80 \%$ of which are acute lymphoblastic leukemias (ALL).

The peak incidence of childhood leukemia is approximately four years of age, although this peak is due almost entirely to patients diagnosed with ALL. Acute myelogenous leukemia (AML) is equally distributed among patients aged 0-10, with a slight increase in incidence in adolescence. ALL is more common among whites than blacks in the US, but AML has an equal distribution among all ethnic groups. The incidence of leukemia is also slightly higher among boys than girls. The ratio of boys with leukemia to girls with leukemia is greatest during adolescence, particularly in the subset of patients with T-cell ALL.

The exact cause of most leukemias is still not known, although there are a number of predisposing factors and exposures. In all likelihood, the cause or causes of most leukemias is multifactorial, including genetic, immune, infectious, and environmental factors.

\section{Clinical Manifestation}

The presenting clinical features of the acute childhood leukemias reflect the uncontrolled proliferation of malignant cells leading to replacement and suppression of normal hematopoietic progenitor cells, and infiltration into extramedullary spaces. Symptoms frequently accumulate in a matter of days or weeks, culminating in some event that brings the child to medical attention. Chronic Myelogenous Leukemia (CML) may present with a more insidious course. The differential diagnosis of an acute leukemia at presentation is included in Table 3.

\section{Classification}

ALL is the most common form of leukemia in childhood (see Figure 1). Subclassification into early pre-B-cell, pre-B-cell, B-cell, and T-cell lymphoblastic leukemias is essential to treatment, and prognostic stratification of patients. The myeloid leukemias can be categorized according to either the cell of origin (French-American-British, FAB, system) or cytogenetic and morphologic features (WHO (World Health Organization) Classification system). The distinguishing phenotypic features of each class of leukemia is listed in Table 4.

Cytogenetic and Molecular Markers

There are several well-described genetic mutations that not only offer insight into the pathogenesis of an acute leukemia, but which significantly influence prognosis.

Treatment of ALL

\section{Risk-Stratified Therapy}

Risk-adapted therapies, taking into account clinical features (age, white blood cell count, and response to induction therapies) and biologic features (ploidy and mutations of known prognostic significance), are used to maximize cure rates while minimizing long-term side effects. Standard risk and high-risk patients are initially distinguished based on age (between 1 and 9.99 years of age is standard risk) and the initial white blood cell count $(\leq 50,000 / \mu \mathrm{L}$ is standard risk). Other clinical features used to stratify therapy include gender, leukemia phenotype, cytogenetics, and response to initial therapy.

Principles of Treatment

Standard therapy is divided into phases of remission induction, consolidation, delayed intensification, extramedullary disease treatment or prophylaxis, and maintenance. The timing, number, and chemotherapy components of each phase may differ between protocols, but the principles of therapy are similar. Remission induction in achieved in most cases with 
Table I: Factors Associated with a Higher Risk of Developing Leukemia

Environmental Exposures

Ionizing radiation (therapeutic or environmental exposure)

Epipodophyllotoxin or alkylator-based chemothrapy

Genetic Predisposition

Siblings of patient with a childhood leukemia

Down syndrome

Fanconi anemia

Bloom syndrome

Ataxia telangiectasia

Paroxysmal nocturnal hemoglobinuria

Congenital hypogammaglobulinemia

Wiskott-Aldrich syndrome

Neurofibromatisis

Klinefelter syndrome

Schwachman syndrome

Table 2: Common Clinical and Laboratory Findings Present at Diagnosis in Children with Leukemia

\begin{tabular}{lcc}
\hline & ALL (\%)(I76) & AML (\%)( I77) \\
\hline Clinical Features & & \\
\hline Fever & $6 I$ & 34 \\
\hline Pallor & 55 & 25 \\
\hline Petechiae, purpura, bleeding & 48 & 33 \\
\hline Anorexia or weight loss & 33 & 22 \\
\hline Fatigue, malaise & 30 & 19 \\
\hline Bone, joint pain & 38 & 18 \\
\hline Lymphadenopathy & 50 & 14 \\
\hline Hepatosplenomegaly & 68 & 55 \\
\hline Swollen gingivae & - & 8 \\
\hline Cough, dysphagia & - & 41 \\
\hline Recurrent infection & - & 3 \\
\hline Neurologic symptoms & 3 & 10 \\
\hline
\end{tabular}

Laboratory Features

\begin{tabular}{lll}
\hline White blood cell count $\left(\right.$ per $\left.\mathrm{mm}^{3}\right)$ & & \\
\hline$<10,000$ & 53 & 39 \\
\hline $10,000-49,000$ & 30 & 29 \\
\hline$>50,000$ & 17 & 32 \\
\hline Hemoglobin $(\mathrm{g} / \mathrm{dl})$ & & 41 \\
\hline$<7$ & 43 & 48 \\
\hline $7-11$ & 45 & 11
\end{tabular}

Platelet count (per $\mathrm{mm}^{3}$ )

\begin{tabular}{lcc}
\hline$<20,000$ & 28 & 15 \\
\hline $20,000-99,000$ & 47 & 67 \\
\hline$>100,000$ & 25 & 18 \\
\hline Coagulopathy & - & 17
\end{tabular}

Adapted from Aesselin BL. Leukemias. In Hoekelman RA, Adam HM, Nelson NM, et. al. Primary Pediatric Care, 4th Ed. Mosby, (200I). $A L L=$ acute lymphoblastic leukemias, $A M L=$ acute myelogenous leukemia three drugs (vincristine, asparaginase, and a corticosteroid) in standard risk patients, or four drugs in high risk patients (the same three as in standard risk patients with the addition of daunorubicin). The goal is to induce a complete remission, defined as the reduction the leukemia cell burden below clinical, morphological, and molecular levels of detection after recovery of normal blood counts. With current chemotherapeutic regimens, remission induction is possible in more than $95 \%$ of children with ALL. Many protocols follow the induction phase, with a consolidation phase heavy in antimetabolites to secure or consolidate the remission. Stratification to higher or lower intensity therapies may occur at the end of induction based on molecular quantification of minimal residual disease.

Delayed intensification is a second phase of intensified chemotherapy similar to the induction and consolidations phases. The addition of a delayed intensification phase by the Berlin-Frankfurt-Munich (BFM) group has led to significant improvement in survival in all childhood ALL patients. Survival among patients with high-risk leukemia may be further improved by augmenting the regimen with a second delayed intensification.

In the past two decades, several trials have demonstrated that central nervous system (CNS) radiation may be reduced (to $1200 \mathrm{cGy}$ to $1800 \mathrm{cGy}$ ) or eliminated in patients with low-risk B-cell ALL with a rapid early response to induction therapy. Elimination of cranial radiation in patients with T-cell ALL has not proven feasible. Reduction in cranial radiation is designed to reduce long-term endocrine and cognitive effects, as well as reduce the risk of secondary malignancies

Unlike treatment paradigms for most malignancies, successful therapy for childhood ALL includes a protracted maintenance period. Attempts to reduce therapy to less than 24 months have never been successful. In current Children's Oncology Group (COG) Studies, boys with standard risk ALL receive three years of postremission therapy, while girls with ALL receive two years of post-remission therapy. The intensity of therapy is greatly reduced during maintenance, and many patients may return to school during this period.

Infant $A L L$

Despite intensified therapy, infants less than six months of age with ALL have an event-free survival of less than $10 \%$, while those older than six months have an eventfree survival of $40 \%$. Currently, all infants still have a higher incidence of relapse, death from toxicity, and long-term side effects when compared to older children. 



\section{Complete or Partial Response in Over $\mathbf{3 0 \%}$ of Heavily Pretreated Patients}

In a phase 2 single arm, open label study in pediatric relapsed/refractory acute lymphoblastic leukemia (ALL) ( $n=49)$, over $30 \%$ achieved a complete or partial response.

All patients had disease that had relapsed after and/or was refractory to two or more prior therapies. Most patients, $46 / 49$ (93.8\%), had received 2 to 4 prior regimens and $15 / 49(30.6 \%)$ of the patients had undergone at least 1 prior transplant

6 of 15 patients who responded to treatment with Clolar were able to undergo post-treatment bone marrow transplantation, and response duration could not be determined. In the 9 responding patients who were not transplanted, response durations were: CR: 43, 50, 82, 93+, and 160+ days; CRp: 32 days; PR: 7, 16, and 21 days.

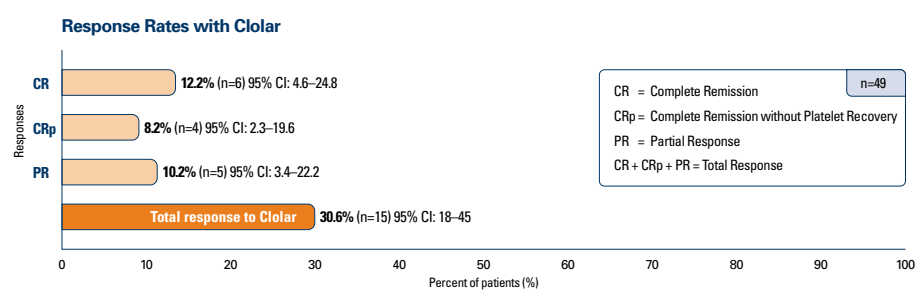

Response rates were determined by an unblinded independent response review panel. Complete Remission (CR) defined as no circulating blasts or extramedullary disease, $<5 \%$ bone marrow blasts, and recovery of peripheral blood counts (platelets $>100 \times 10^{\%} / \mathrm{L}$ and absolute neutrophil count $\left[\right.$ ANC] $>1.0 \times 10^{9} / \mathrm{L}$ ). Complete Remission in the Absence of Total Platelet Recovery (CRp) defined as meeting all criteria of CR except for recovery of platelet counts to $>100 \times 10^{9} / \mathrm{L}$. Partial Response (PR) defined as no circulating blasts, $>5 \%$ and $<25 \%$ bone marrow blasts, and appearance of normal progenitor cells.
Clolar is indicated for the treatment of pediatric patients 1 to 21 years old with relapsed or refractory acute lymphoblastic leukemia after at least two prior regimens. This use is based on the induction of complete responses. Randomized trials demonstrating increased survival or other clinical benefit have not been conducted.

\section{Tolerability}

Clolar should be administered under the supervision of a qualified physician experienced in the use of antineoplastic therapy. Suppression of bone marrow function, which is usually reversible and dose dependent, should be anticipated and is likely to increase the risk of infection, including severe sepsis. Administration of Clolar results in a rapid reduction in peripheral leukemia cells. Patients should be evaluated and monitored for signs and symptoms of tumor lysis syndrome and cytokine release (e.g., tachypnea, tachycardia, hypotension, pulmonary edema) that could develop into systemic inflammatory response syndrome (SIRS)/capillary leak syndrome, and organ dysfunction. Clolar should be discontinued immediately in the event of clinically significant signs or symptoms of SIRS or capillary leak syndrome, either of which can be fatal, and use of steroids, diuretics, and albumin considered.

The most common adverse effects after Clolar treatment, regardless of causality, were gastrointestinal tract symptoms, including vomiting (grade 3: $8 \%$; grade $4: 1 \%$ ), nausea (grade 3: 15\%; grade 4: 1\%), and diarrhea (grade 3: 10\%); hematologic effects, including anemia (grade 3: 70\%; grade 4: 18\%), leukopenia (grade 4: 99\%), thrombocytopenia (grade 3: $36 \%$; grade 4: $64 \%$ ), neutropenia (grade 3: $3 \%$; grade 4: $7 \%$ ), and febrile neutropenia (grade 3: 53\%; grade 4: 3\%); and infection (grade 3: 56\%; grade 4: 18\%).

Hepato-biliary toxicities were frequently observed in pediatric patients during treatment with Clolar. The most frequently reported cardiac disorder was tachycardia (34\%), which was, however, already present in $27.4 \%$ of patients at study entry. Left ventricular systolic dysfunction was also noted. Since Clolar is excreted primarily by the kidneys, drugs with known renal toxicity should be avoided during the 5 days of Clolar administration. In addition, since the liver is a known target organ for Clolar toxicity, concomitant use of medications known to induce hepatic toxicity should also be avoided.

Severe bone marrow suppression, including neutropenia, anemia, and thrombocytopenia, have been observed in patients treated with Clolar. Because of the pre-existing immunocompromised condition of these patients and prolonged neutropenia that can result from treatment with Clolar, patients are at increased risk for severe opportunistic infections.

Pericardial effusion was a frequent finding in these patients on post-treatment studies Careful hematologic monitoring during therapy is important. Hepatic and renal function should be assessed prior to and during treatment with Clolar, as the liver is a target organ for Clolar toxicity and the kidneys are the predominant mode of Clolar excretion.

Patients receiving Clolar may experience vomiting and diarrhea; they should therefore be advised regarding appropriate measures to avoid dehydration. Clolar may cause fetal harm when administered to a pregnant woman. Women of childbearing potential should be advised to avoid becoming pregnant and avoid breastfeeding while receiving treatment with Clolar.

Please see brief summary of full prescribing information on adjacent page.

Respond now.

800-RX-CLOLAR or visit www.clolar.com
(C2006 Genzyme Corporation. All rights reserved.

Clolar is a registered trademark and A Chance to Respond is a trademark of Genzyme Corporation. CLO/US/P006a/0406 


\section{Brief Summary of Full Prescribing Information}

\begin{tabular}{|c|}
\hline \\
\hline A chance to respond \\
\hline
\end{tabular}

INDICATIONS AND USAGE

CLOLAR ${ }^{\circ}$ is indicated for the treatment of pediatric patients 1 to 21 years old with relapsed or refractory acute Iymphoblasti the induction of completer responsses. Randomized trials demon-

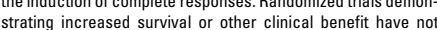
strating increased
been conducted.

\section{CONTRAINDICATIONS}

None

WARNINGS

CLOLAR should be administered under the supervision of a qualified physician experienced in the use of antineoplastic therapy. Suppression of bone marrow function should be anticipated. This is usually reversible and appears to be dose dependent. The use of CLOLAR ${ }^{\circ}$ is likely to increase the risk of infection, incluading severe sepsis, as a result of bone marrow



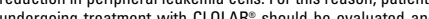



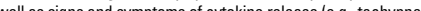
tachycardis hypotension pulmory edemal that coud develo inte systemic inflammotory response syndrome SIRS/cailloy leak syndrome, and organ dystunction. Physicins are encouraged to give, continuous IV fluids throughout the five days of CLOLAR administration to reduce the effects of tumor lysis and other adverse events. Allopurinol should be administered hyperuricemia is expected, CLOLAR ${ }^{\circ}$ should be discontinued immediately in the event of clinically significant signs or symptoms of SIRS or capililary leak syndrome, either of which can fatal, and use of steroids, diuretics, and albumin considered. CLLLAR" can be re-instituted when the patient is stable, generally at a lower dose.

Severe bone marrow suppression, including neutropenia, anemia, and thrombocytopenia, has been observed in patients treated with CLOLAR. A A initiation of treatment, most patients in the clinical studies had hematological impairment as a manfestation of leukemia. Because of the pre-existing in compromised condition of these patients and prolonged neu-
tropenia that can result from treatment with CLOLAR are at increased risk for severe opportunistic infections. Careful hematological monitoring during therapy is important and hepatic and renal function should be assessed prior to and during treatment with CLLLAR because of CLOLAR"s predominantly renal excretion and because the liver is a target organ



\section{Hepatic and Renal Impairment}

CLOLAR ${ }^{\circledast}$ has not been studied in patients with hepatic or rena dysfunction. Its use in such patients should be undertaken only with the greatest caution.

Pregnancy - Teratogenic Effects: Pregnancy Category D CLOLAR ${ }^{\infty}$ (clofarabine) may cause fetal harm when administered to a pregnant woman.

Clofarabine was teratogenic in rats and rabbits. Developmental toxicity (reduced fetal body weight and increased post-implantation loss) and increased incidences of malformations and variations (gross external, soft tissue, skeletal and retarded ossification) were observed in rats receiving $54 \mathrm{mg} / \mathrm{m}^{2} / \mathrm{day}$ (approximately equivalent to the recommended clinical dose on a $\mathrm{mg} / \mathrm{m}^{2}$ basis), and in rabbits receiving $12 \mathrm{mg} / \mathrm{m}^{2} / \mathrm{day}$ (approximatel
$23 \%$ of the recommended clinical dose on a $\mathrm{mg} / \mathrm{m}^{2}$ basis).

There are no adequate and well-controlled studies in pregnant women using clofarabine. If this drug is used during pregnancy, or if the patient becomes pregnant while taking this drug, the
patient should be apprised of the potential hazard to the fetus. Women of childbearing potential should be advised to avoid becoming pregnant while receiving treatment with clofarabine. PRECAUTIONS

\section{Information for Patients and Caregivers}

Physicians are advised to discuss the following with patients to whom CLOLAR ${ }^{\circledast}$ will be administered and patient caregivers, as appropriate.

\section{Dehydration/Hypotension}

Patients receiving CLOLAR ${ }^{\circledR}$ may experience vomiting and diarrhea; they should therefore be advised regarding appropriate measures to avoid dehydration. Patients should be instructed to seek medical advice if they experience symptoms of dizziness, lightheadedness, fainting spells, or decreased urine output. CLOLAR ${ }^{\circledast}$ administration should be stopped if the patient develops hypotension for any reason during the 5 days of administration. If hypotension is transient and resolves without pharmaco-
logical intervention, CLOLAR generally at a lower dose.

\section{Concomitant Medications}

Since CLOLAR is excreted primarily by the kidneys, drugs with known renal toxicity should be avoided during the 5 days of CLOLAR administration. In addition, since the liver is a known ions known to induce heptic toxicity should also be auded. tions known to induce hepatic toxicity should also be avoided. cardiac function should be closely monitored during administration of CLOLAR
Pregnancy/Nursing

All patients should be advised to use effective contraceptive measures to prevent pregnancy. Female patients should be
advised to avoid breast-feeding during treatment with CLOLAR

\section{Laboratory Tests}

Complete blood counts and platelet counts should be obtained at regular intervals during CLOLAR $R^{\circ}$ therapy, and more frequently in patients who develop cytopenias. In addition, liver and kidney function should be monitored frequently during the 5 days of CLOLAR ${ }^{\circledast}$ administration

\section{Drug Interactions}

Although no clinical drug-drug interaction studies have been conducted to date, on the basis of the in vitro studies, cytochrome p450 inhibitors and inducers are unlikely to affect metabolism of cytochrome $\mathrm{p} 450$ substrates has not been studied.

\section{Drug/Laboratory Tests Interactions}

There are no known clinically signific ant interactions of CLOLAR with other medications or laboratory tests. No formal drug/lab-
oratorytestinteraction studies have been conducted with $C$ COLAR

\section{Carcinogenesis, Mutagenesis, Impairment of Fertility}

Carcinogenesis

Clofarabine has not been tested for carcinogenic potential.

Mutagenesis

Clofarabine showed clastogenic activity in the in vitro mammalian cell chromosome aberration assay (CHO cells) and in the in vivo rat micronucleus assay. It did not show evidence of

Impairment of Fertility

Studies in mice, rats, and dogs have demonstrated dose-related adverse effects on male reproductive organs. Seminiferous tubule and testicular degeneration and atrophy were reported in male mice receiving intraperitoneal (IP) doses of $3 \mathrm{mg} / \mathrm{kg} / \mathrm{day}$ $19 \mathrm{mg} / \mathrm{m}^{2} / \mathrm{day}$, approximately $17 \%$ of the recommended clinical dose on a mg/ $/ \mathrm{m}^{2}$ basis). The testes of rats receiving $25 \mathrm{mg} / \mathrm{kg} / \mathrm{day}$ $\left(150 \mathrm{mg} / \mathrm{m}^{2} /\right.$ day, approximately 3 times the recommended clinical dose on a $\mathrm{mg} / \mathrm{m}^{2}$ basis) in a 6 -month IV study had bilateral degeneration of the seminiferous epithelium with retained spermatids and atrophy of interstitial cells. In a 6-month IV dog study, cell degeneration of the epididymis and degeneration of the seminiferous epithelium in the testes were observed in dogs receiving $0.375 \mathrm{mg} / \mathrm{kg} / \mathrm{day}\left(7.5 \mathrm{mg} / \mathrm{m}^{2} / \mathrm{day}\right.$, approximately $14 \%$ of the recommended clinical dose on a $\mathrm{mg} / \mathrm{m}^{2}$ basis). Ovarian atro-
phy or degeneration and uterine mucosal apoptosis were phy or degeneration and uterine mucosal apoptosis were
observed in female mice at $75 \mathrm{mg} / \mathrm{kg} /$ day $\left(225 \mathrm{mg} / \mathrm{m}^{2} / \mathrm{day}\right.$, observed in female mice at $75 \mathrm{mg} / \mathrm{kg} / \mathrm{day}\left(225 \mathrm{mg} / \mathrm{m}^{2} / \mathrm{day}\right.$, approximately 4-fold of the recommended human dose on The effect on human fertility is unknown.

\section{Pregnancy}

Teratogenic Effects: Pregnancy Category D

See WARNINGS.

Nursing Mothers

It is not known whether clofarabine or its metabolites are excreted in human milk. Because of the potential for tumorigenicity shown for clofarabine in animal studies and the potental for serious adverse reactions, women treated with clofara-

Other Special Population: Adults

Safety and efficacy have not been established in adults. One study was performed in highly refractory and/or relapsed adult patients with hematologic malignancies. The Phase 2 dose of CLOLAR ${ }^{x}$ was determined to be $40 \mathrm{mg} / \mathrm{m}^{2} /$ day administered as a 1- to 2-hour IVI daily $x 5$ every 28 days.

\section{ADVERSE REACTIONS}

One hundred thirteen (113) pediatric patients with ALL (67) o AML (46) were exposed to CLOLAR

Ninety six (96) of the pediatric patients treated in clinical trials The most common adverse effects after CLOLAR ${ }^{\infty}$ treatment, The most common adverse effects after CLOLAR
regardless of causality, were gastrointestinal tract symptoms, regardless of causality, were gastrointestinal tract symptoms,
including vomiting, nausea, and diarrhea; hematologic effects, including vomiting, nausea, and diarrhea; hematologic effects,
including anemia, leukopenia, thrombocytopenia, neutropenia, ancluding anemia, leukopenia, throm neutropenia; and infection.

Table 1 lists adverse events by System Organ Class regardless of causality, including severe or life-threatening events (NC CTC grade 3 or grade 4), reported in $\geq 10 \%$ of the 96 patients follow-up of certain events is given below.

Table 1: Most Commonly Reported ( $\geq 10 \%$ Overall) Adverse Events by System Organ Class ( $N=96$ )

\begin{tabular}{|c|c|c|c|c|c|c|}
\hline \multirow{3}{*}{$\begin{array}{l}\text { System Organ Class } \\
\text { Adverse Event' }\end{array}$} & \multicolumn{6}{|c|}{$52 \mathrm{mg} / \mathrm{m}^{2}(\mathrm{~N}=96)$} \\
\hline & \multicolumn{2}{|c|}{\begin{tabular}{|l|} 
Total \\
\end{tabular}} & \multicolumn{2}{|c|}{ Grade 3 } & \multicolumn{2}{|c|}{ Grade 4} \\
\hline & $\mathbf{N}$ & $\%$ & $\mathbf{n}$ & $\%$ & & $\%$ \\
\hline \multicolumn{7}{|c|}{ Blood and Lymphatic System Disorders } \\
\hline Febrile neutropenia & 55 & 57 & 51 & 53 & 3 & 3 \\
\hline Neutropenia & 10 & 10 & 3 & 3 & 7 & 7 \\
\hline Transfusion reaction & 10 & 10 & 3 & 3 & & . \\
\hline \multicolumn{7}{|l|}{ Cardiac Disorders } \\
\hline Tachycardia NOS & 33 & 34 & 6 & 6 & & . \\
\hline \multicolumn{7}{|c|}{ Gastrointestinal Disorders } \\
\hline Abdominal pain NOS & 35 & 36 & 7 & 7 & . & . \\
\hline Constipation & 20 & 21 & , & 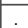 & . & . \\
\hline Diarrhea NOS & 51 & 53 & 10 & 10 & & \\
\hline Gingival bleeding & 14 & 15 & 7 & 7 & 1 & 1 \\
\hline Nausea & 72 & 75 & 14 & 15 & 1 & 1 \\
\hline Sore throat NOS & 13 & 14 & & & & . \\
\hline Vomiting NOS & 80 & 83 & 8 & 8 & 1 & 1 \\
\hline
\end{tabular}

Table 1: Most Commonly Reported ( $\geq 10 \%$ Overall) Adverse Events by System Organ Class ( $\mathrm{N}=96$ ) (Continued)

\begin{tabular}{|c|c|c|c|c|c|c|}
\hline \multirow{3}{*}{$\begin{array}{l}\text { System Organ Class } \\
\text { Adverse Event' }\end{array}$} & \multicolumn{6}{|c|}{$52 \mathrm{mg} / \mathrm{m}^{2}(\mathrm{~N}=96)$} \\
\hline & \multicolumn{2}{|c|}{ Total } & \multicolumn{2}{|c|}{ Grade 3} & \multicolumn{2}{|c|}{ Grade 4} \\
\hline & & $\%$ & $\mathrm{n}$ & $\%$ & $\mathrm{n}$ & $\%$ \\
\hline \multicolumn{7}{|c|}{ General Disorders and Administration Site Conditions } \\
\hline Edema NOS & 19 & 20 & 1 & 1 & 2 & 2 \\
\hline Fatigue & 35 & 36 & 3 & 3 & 1 & 1 \\
\hline Injection site pain & 13 & 14 & 1 & 1 & & \\
\hline Lethargy & 11 & 11 & . & . & & \\
\hline Mucosal inflammation NOS & 17 & 18 & 3 & 3 & & \\
\hline Pain NOS & 18 & 19 & 6 & 6 & 1 & 1 \\
\hline Pyrexia & 39 & 41 & 15 & 16 & & \\
\hline Rigors & 36 & 38 & 3 & 3 & & \\
\hline \multicolumn{7}{|l|}{ Hepato-Biliary Disorders } \\
\hline Hepatomegaly & 14 & 15 & 8 & 8 & & . \\
\hline Jaundice NOS & 14 & 15 & 2 & 2 & & \\
\hline \multicolumn{7}{|l|}{ Infections and Infestations } \\
\hline Bacteremia & 10 & 10 & 10 & 10 & & \\
\hline Cellulitis & 11 & 11 & 9 & 9 & & \\
\hline Herpes simplex & 11 & 11 & 6 & 6 & & \\
\hline Oral candidiasis & 12 & 13 & 2 & 2 & & \\
\hline Pneumonia NOS & 10 & 10 & 5 & 5 & 2 & 2 \\
\hline Sepsis NOS & 14 & 15 & 7 & 7 & 7 & 7 \\
\hline
\end{tabular}

\begin{tabular}{|l|c|ccc|c|}
\hline Staphylococcal infection NOS & 12 & 13 & 10 & 10 & . \\
\hline
\end{tabular}

\begin{tabular}{|l|l|l|l|l|l|l|}
\hline Investigations & 10 & 10 & 1 & 1 &. &. \\
\hline Weight decreased & &
\end{tabular}

Metabolism and Nutrition Disorders

\begin{tabular}{|l|l|l|l|l|l|l|}
\hline Anorexia & 30 & 31 & 5 & 5 & 7 & 7 \\
\hline
\end{tabular}

\begin{tabular}{|l|lllllll|}
\hline Appetite decreased NOS & 11 & 11 &. &. & &. & . \\
\hline Musculoskeletal, Connective Tissue and Bone Disorders \\
\hline A
\end{tabular}

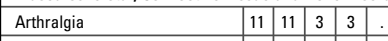

\begin{tabular}{|l|c|c|c|c|c|c|}
\hline Arthralgia & 11 & 11 & 3 & 3 &. & . \\
\hline Back pain & 12 & 13 & 3 & 3 &. & . \\
\hline Myalgia & 13 & 14 &. &. &. &. \\
\hline Pain in limb & 28 & 29 & 5 & 5 &. & . \\
\hline Neras
\end{tabular}

Pain in limb

Nervous System Disorder

Dizziness (except vertigo)

Headache NOS

Somnolence

\begin{tabular}{|l|l|l|}
\hline 15 & 16 \\
\hline 44 & 46 & \\
\hline
\end{tabular}

Tremor NEC

Psychiatric Disorders

Anxiety NEC

Depression NEC

Irritability

Renal and Urinary Disorders

Hematuria

Respir

\begin{tabular}{|l|c|c|c|c|c|c|}
\hline Cough & 18 & 19 &. &. &. &. \\
\hline Dyspnea NOS & 12 & 13 & 4 & 4 & 2 & 2 \\
\hline Epistaxis & 30 & 31 & 14 & 15 &. &. \\
\hline Pleural effusion & 10 & 10 & 3 & 3 & 2 & 2 \\
\hline Respiratory distress & 13 & 14 & 6 & 6 & 5 & 5 \\
\hline \multicolumn{2}{|c|}{ Skin and Subcutaneous Tissue Disorders } \\
\hline Contusion & 11 & 11 & 1 & 1 & \multicolumn{5}{|l|}{} \\
\hline Dermatitis NOS & 39 & 41 & 7 & 7 &. &. \\
\hline Dry skin & 10 & 10 & 1 & 1 &. &. \\
\hline Erythema NEC & 17 & 18 &. &. &. &. \\
\hline $\begin{array}{l}\text { Palmar-plantar } \\
\text { erythrodysesthesia syndrome }\end{array}$ & 12 & 13 & 4 & 4 &. & \\
\hline Petechiae & 28 & 29 & 7 & 7 &. &. \\
\hline Pruritus NOS & 45 & 47 & 1 & 1 &. &. \\
\hline Vascular Disorders & \multicolumn{7}{|c|}{} \\
\hline Flushing & 17 & 18 &. &. &. &. \\
\hline Hypertension NOS & 11 & 11 & 4 & 4 &. &. \\
\hline Hypotension NOS & 28 & 29 & 12 & 13 & 7 & 7 \\
\hline
\end{tabular}

Pripeng

1 Patients with mo.
counted only once.

Grade 4 includes deaths (Grade 5 .

\section{Cardiovascular}

The most frequently reported cardiac disorder was tachycardia $(34 \%)$, which was, however, already present in $27.4 \%$ of patients at study entry. Most of the cardiac adverse events were reported in the first 2 cycles. Pericardial effusion was a frequent finding in these patients on post-treatment studies,
$[19 / 55(35 \%)]$. The effusion was almost always minimal to small $[19 / 55(35 \%)]$. The effusion was almost always mi
and in no cases had hemodynamic significance.

Left ventricular systolic dysfunction (LVSD) was also noted Fifteen out of fifty-five patients [15/55 (27\%)] had some evidence of LVSD after study entry. In most cases where subsequent follow-up data were available, the LVSD appeared to be transient.
The exact etiology for the LVSD is unclear because of previous therapy or serious concurrent illness.

\section{Hepatic}

Hepato-biliary toxicities were frequently observed in pediatric patients during treatment with CLOLAR ${ }^{\varpi}$. Grade 3 or 4 elevated aspartate aminotransferase (AST) occurred in $38 \%$ of patients occurred in $44 \%$ of patients. Grade 3 or 4 elevated bilirub occurred in $15 \%$ of patients, with 2 cases of grade 4 hyperbilirubinemia resulting in treatment discontinuation.

For patients with follow-up data, elevations in AST and ALT were transient and typically of $<2$ weeks duration. The majority

administration and returned to baseline or $\leq$ grade 2 within several days. Although less common, elevations in bilirubin ap eared to be more persistent. Where follow-up data are available, the median time to recovery from grade 3 and grade 4 elevations in bilirubin to $\leq$ grade 2 was 6 days.

Infection

At baseline, $47 \%$ of the patients had 1 or more concurrent infections. A total of $85 \%$ of patients experienced at least 1 infection after Renal

The most prevalent renal toxicity was elevated creatinine Grade 3 or 4 elevated creatinine occurred in $6 \%$ of patients. Nephrotoxic medications, tumor lysis, and tumor lysis with hyperuricemia may contribute to renal toxicity.

Systemic Inflammatory Response Syndrome (SIRS)/ Capillary Leak Syndrome

Capillary leak syndrome or SIRS (signs and symptoms of cytokine release, e.g., tachypnea, tachycardia, hypotension, pulmonary edema) occurred in 4 pediatric patients overall (3 ALL, 1 AML). Several patients developed rapid onset of respiratory distress, hypotension, capillary leak (pleural and pericardial effusions), and multi-organ failure. Close monitoring for this syndrome and early intervention are recommended. The use of prophylactic steroids (e.g., $100 \mathrm{mg} / \mathrm{m}^{2}$ hydrocortisone on Days 1 through 3) may be of benefit in preventing signs or symptoms of SIRS or capillary leak. Physicians should be alert to early indications of this syndrome and should immediately discontinue
CLOLAR CLOLAR administration if they occur and provide appropriate
supportive measures. After the patient is stabilized and organ supportive measures. After the patient is stabilized and organ
function has returned to baseline, re-treatment with CLOLAR function has returned to baseline, re
can be considered at a lower dose.

\section{Overdosage}

There were no known overdoses of CLOLAR ${ }^{\circ}$. The highest daily dose administered to a human to date (on a $\mathrm{mg} / \mathrm{m}^{2}$ basis) has been $70 \mathrm{mg} / \mathrm{m}^{2} /$ day $\times 5$ days ( 2 pediatric ALL patients). The toxicities grade 2 and 3 vomiting, and grade 3 maculopapular rash.
.

\section{DOSAGE AND ADMINISTRATION}

\section{Recommended Dose}

CLOLAR $^{\circ}$ should be diluted per instructions below with $5 \%$ Dextrose Injection, USP, or $0.9 \%$ Sodim Chloride Iniection, USP,

The recommended pediatric dose and schedule is $52 \mathrm{mg} / \mathrm{m}^{2}$ administered by intravenous infusion (IVI) over 2 hours daily for 5 consecutive days. Treatment cycles are repeated following recovery or return to baseline organ function, approximately every 2 to 6 weeks. The dosage is based on the patient's body surface area (BSA), calculated using the actual height and weight before the start of each cycle. To prevent drug incom-
patibilities, no other medications should be administered patibilities, no other medications
through the same intravenous line.

CLOLAR ${ }^{\circ}$ has not been studied in patients with hepatic or renal dysfunction. Its use in such patients should be undertaken only with the greatest caution.

Physicians are encouraged to give continuous IV fluids throughout the 5 days of CLOLAR administration to reduce the effects of tumor lysis and other adverse events. The use of prophylactic steroids (e.g., $100 \mathrm{mg} / \mathrm{m}^{2}$ hydrocortisone on Days 1 through 3) may be of benefit in preventing signs or symptoms of SIRS or capillary leak (e.g., hypotension). If patients show early signs or symptoms of SIRS or capillary leak (e.g., hypotension),
the physician should immediately discontinue CLOLAR adminthe physician should immediately discontinue CLOLAR ${ }^{\circ}$ admin-
istration and provide appropriate supportive measures. Close istration and provide appropriate supportive measures. Close
monitoring of renal and hepatic function during the 5 days of
CLOLAR administration is advised. If substantial increases in 
Infants under six months old, with abnormalities involving 11q23, appear to be at highest risk.

Treatment of AML

Principles of Therapy

The event-free survival rate in childhood AML has reached a plateau at 60\%, despite aggressive intensification of therapy. Cytarabine and daunorubicin, in combination with either etoposide or thioguanine, will induce a complete remission in more than $90 \%$ of patients. Attempts to substitute idarubicin or mitoxantrone for daunorubicin, or increase the dose of cytarabine, have largely proven to be of comparable efficacy, and, occasionally, greater toxicity. Intensive post-remission therapies have been developed using multi-agent regimens cycled every four to six weeks. There have been no randomized, head-to-head comparisons of the different regimens, but none have succeeded in improving long-term, event-free survival beyond $60 \%$. Most studies of allogeneic transplant in children with AML non-randomly assign patients with matched sibling donors to undergo transplant, and those without sibling donors to continue with conventional chemotherapy. Relapse-free survival is generally better in the patients who undergo transplantation, but overall survival is comparable due to transplant-related mortality.

Acute Promyelocytic Leukemia

Patients with acute promyelocytic leukemia (APML) do not do well with conventional AML therapy. Rather, regimens intensifying the anthracycline dose in induction and incorporating retinoic acid, mercaptopurine, and methotrexate into maintenance therapy have led to five-year survival rates exceeding 80\% among children with APML.

\section{CML}

In children and adults, CML is characterized by the presence of the Philadelphia $(\mathrm{Ph})$ chromosome. Until recently, standard therapy for CML in children and adults included hydroxyurea, interferon, and low-dose cytarabine. While clinical improvements in hematologic parameters were possible with these therapies, cytogenetic remission were infrequent. More recently, imatinib mesylate was developed as a specific inhibitor of the BCR-ABL (breakpoint cluster regionAbelson) tyrosine kinase. Results from recent clinical trials have rapidly established imatinib as the standardof-care for CML, and cytogenetic remissions are now expected. Limited data are available on imatinib mesylate in children, but preliminary data are
Table 6: European Group for the Immunological Characterization of Leukemias Scoring System

\begin{tabular}{lllr}
\hline Points & B-Cell ALL & T Cell ALL & AML \\
\hline 2 & cyCD79 & cyCD3 or memCD3 & MPO \\
& cyCD22 & Anti-TCR & \\
& cylgM & & \\
\hline CD/9 & $C D 2$ & $C D / 17$ \\
& $C D 20$ & $C D 5$ & $C D / 3$ \\
& $C D 10$ & $C D 8$ & $C D 33$ \\
& & $C D / 0$ & $C D 65$ \\
\hline 0.5 & $T d T$ & $T d T$ & $C D / 4$ \\
& $C D 24$ & $C D 7$ & $C D / 5$ \\
\hline
\end{tabular}

$c y=$ cytoplasmic, $m e m=$ membrane, $T C R=T$ cell receptor, $M P O=$ myeloperoxidase,$T d T=$ terminal deoxynucleotidyl transferase

promising. Imatinib mesylate has dramatically changed therapy for CML, but it is still not a cure. The only proven curative strategy for children with CML is allogeneic stem cell transplantation (SCT). Survival after SCT for CML is reported to be $70-80 \%$ with a matched sibling donor and 40-60\% when unrelated donors are used.

Relapsed Leukemias and STC

$A L L$

Despite development of successful therapies in childhood leukemias, 20-25\% of children with ALL and $50 \%$ of children with AML will relapse after chemotherapy treatment. In fact, more children are diagnosed with relapsed leukemia than Hodgkin's disease (HD) and pediatric sarcomas. In most children with relapsed leukemia, long-term, event-free survival is less than 50\%, even with SCT. Remission re-induction following relapse is relatively good, but overall survival remains poor, owing to a deficiency in effective therapies. The three-year, event-free survival for patients with a first remission less than 18 month is 4\%; 18-30 months, 10\%; and greater than 30 months, 41\%. Most events occur within six months of relapse and fewer than half of the patients assigned to unrelated donor transplants actually made it to transplant in second remission. These results are striking and speak strongly to the need for additional therapies for ALL.

\section{$A M L$}

As is the case with ALL, hematologic relapse remains the most common adverse event in patients with AML. About $30-40 \%$ of patients who achieve a remission will relapse, while $25 \%$ will have residual disease after intensive induction therapy. Remission re-induction rates for all patients with AML in first relapse ranges from $50-80 \%$ using multiagent chemotherapy. Overall survival following relapse is a dismal $30-40 \%$. 
Figure I:Types and Frequencies of Leukemias in Children

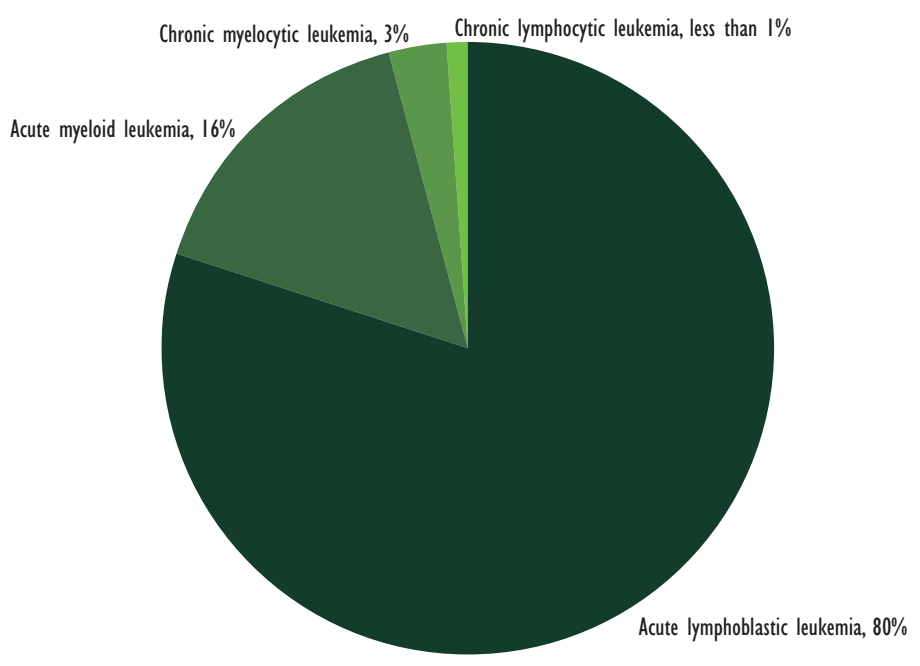

New Agents

As high-dose chemotherapy with SCT is not enough for high-risk, relapsed, and refractory leukemias, new agents are clearly needed. One of the driving forces in the identification, rational synthesis, and development of new agents-many of which are the prototypes in entirely new classes of antineoplastics - is an improved understanding of the biological mechanisms involved in leukemogenesis.

Next Generation Cytotoxic Therapies

One strategy employed in new agent development is the encapsulation of proven agents in liposomes containing polyethylene glycol (PEG). Pegylated forms of doxorubicin, vincristine, all-trans retinoic acid (ATRA), and asparaginase are available. Pegylation increases the half-life, enhances tumor delivery of the compounds, decreases the vesicant effect of doxorubicin and vincristine, decreases the cardiac toxicity of doxorubicin, and reduces the number of intramuscular injections of asparaginase .

Cytarabine is a purine nucleoside analog with an excellent track record in pediatric leukemias, both AML and ALL. Fludarabine, cladribine, and clofarabine are nucleoside analogs developed more recently that are also effective in leukemias and have more favorable pharmacologic features than cytarabine. Based on a response rate of $31 \%$ (12\% complete remission), clofarabine was recently approved for use in relapsed ALL in children. This the first drug approved for such an indication in the US for more than two decades. Like cladribine and fludarabine, clofarabine may be used effectively in combination with cytarabine. As a potent inhibitor of ribonucleotide reductase, clofarabine may decrease intracellular deoxynucleotides, decreasing feedback inhibition of deoxycytidine (dCyd) kinase, and thus allowing for an increase in the accumulation of ara-CTP.

Nelarabine (compound 506U78) is a pro-drug of cytarabine. Early studies demonstrated that nelarabine is selectively toxic to lymphoblasts with an early T-cell phenotype. Initial Phase I and II trials conducted by the COG demonstrated activity with an overall response rate of $55 \%$ in first relapse, $25 \%$ in second relapse, and a few responses in third relapse. Unfortunately, there was significant neurotoxicity, including ataxia, confusion, and coma. The effect appears dose-dependent, and the incidence and severity decreased with dose deescalation. Nelarabine is currently being tested by the COG in the backbone of an intensive BFM regimen for patients with newly diagnosed T-cell ALL.

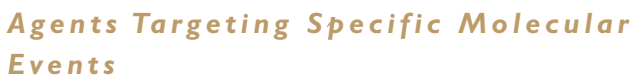

The remarkable success of imatinib mesylate in patients with CML demonstrated the potential of targeted therapies.

Tyrosine Kinase - results from recent clinical trials have rapidly established imatinib as the standard-of-care for CML. A recent phase III prospective trial of $>1,000$ patients conclusively demonstrated that imatinib is superior to interferon alpha (IFN- $\alpha$ ) plus low-dose cytarabine in inducing cytogenetic disease remission and prolonging progression-free survival in adult patients with chronic-phase CML.

Dasatinib-(BMS-354825) is a potent inhibitor of Abl and Src (sarcoma) kinase activity. Imatinib resistance is frequently mediated by mutations in the Abl kinase domain. Unlike imatinib, dasatinib has affinity for all mutations of the Abl kinase domain. In a phase I trial of dasatinib in 36 imatinib-resistant (or intolerant) patients with CML in chronic phase, a complete hematologic response is reported in $86 \%$ of the patients, and a complete cytogenetic response by three months is reported in $28 \%$ of patients. These compounds will have affinity for other tyrosine kinases (e.g. Src, c-kit, plateletderived growth factor receptor (PDGFR)) involved in the pathogenesis of all leukemias. However, it is not yet known what the clinical applications of these compounds will be when targeting non-Abl tyrosine kinases.

Thalidomide and Lenalidomide - thalidomide belongs to the class of compounds called imids. While the mechanism of action is largely unknown, imids do appear to inhibit cytokine production and angiogenesis, while enhancing stromal cell function. Thalidomide experienced a revival 
after demonstrating a $66 \%$ response rate in patients with multiple myeloma. Lenalidomide, is a synthetic compound with less neurotoxicity and teratogenicity. An $83 \%$ response rate is reported among patients with myelodysplastic syndrome (MDS) due to 5(q-).

Proteosome Inhibitors-proteosome inhibitors, like bortezomib, inhibit the breakdown of proteins through the ubiquitin-proteasome pathway. Inhibitors of cell cycle progression and the transcription factor NF-_B accumulate in cells treated with a bortezomib. In an open-label trial of patients with multiple myeloma, a $28 \%$ response rate is reported. In a subsequent phase III randomized trial comparing bortezomib to high-dose dexamethasone, a superior response rate is seen in the patients treated with bortezomib. A phase I trial has been completed by the COG, and phase II combinations are underway.

Arsenic Trioxide - as single agent therapy, arsenic trioxide may induce a complete remission in 90\% of patients with APML. Success of this agent in APML has prompted multiple on-going studies in MDS, myeloma and AML.

Inhibitors of $m$ TOR - the mammalian target of rapamycin (mTOR) is a serine-threonine kinase involved in the activation of Akt and downstream pathways. Rapamycin, an mTOR inhibitor, has demonstrated significant clinical responses in a few patients with relapsed and refractory AML.

Farnesyltransferase inhibitors - ras mutations are common in AML and MDS, and targeting Ras farnesylation is a novel approach currently under investigation. Tipifarnib (R115777) is a farnesyltransferase inhibitor with initial clinical responses in 10 out of 34 elderly patients with AML. Subsequent trials in patients with MDS, CML, and high risk AML were less encouraging. However, additional studies evaluating combinations with other agents are on-going.

Targeting Gene Expression

Histone acetylation/deacetylation and deoxyribonucleid acid (DNA) methylation are epigenetic phenomena that can effect gene expression. Chromatin remodeling agents targeting DNA methylation and histone acetylation are at present in clinical trials. Currently, there are two hypomethylating agents with activity in hematologic malignancies. 5-azacytidine is US Food and Drug Administratioin (FDA)-approved for adults with MDS and 5-aza-2'-dexoycytidine (decitibine) is currently in clinical trials in adults with MDS. Suberoylanilide hydroxamic acid (SAHA), high-dose valproate, and sodium butyrate are histone deacetylase inhibitors. Early investigations into these agents alone and in combination with hypomethylating agents are under way.

Monoclonal Antibodies

Rituximab is a chimeric monoclonal antibody that recognizes the CD20 antigen expressed on mature and immature B-cells. CD20 is expressed in some B-cell ALLs and B-cell non-Hodgkin's lymphoma. Initial studies demonstrated that the addition of rituximab to the standard CHOP (cyclophosphamide, doxorubicin, vincristine, and prednisone) backbone, enhanced survival among patients with B-cell lymphomas. Alemtuzumab is a monoclonal antibody that recognizes CD52 expressed on T-cells, some B-cells and NK cells. Epratuzumab is a humanized monoclonal antibody directed against CD22, an antigen expressed on mature and immature B-cells. In a phase I/II trial of epratuzumab in adult patients with lymphoma, there was a $33 \%$ response rate.

Gemtuzumab ozogamicin (GO) is a humanized monoclonal antibody, directed against the myeloid antigen CD33, conjugated with the cytotoxic agent calicheamicin. It is approved for use in adults with AML over age 60 years of age. In a phase I trial in pediatric patients GO demonstrated a $28 \%$ response rate. However, six of the 13 patients who later underwent SCT developed veno-occlusive disease (VOD). Following promising phase I results, the COG is currently evaluating the role of GO in the backbone of a daunorubicin, etoposide, and cytarabine induction. Humanized monoclonal antibodies conjugated to radioisotopes have also been developed.

\section{Conclusions}

Treatment of childhood leukemias is widely touted as a great success in oncology clinical trials, largely due to the continued incremental increases in long-term survival in children with ALL. Significant challenges still lie ahead as we define new strategies for treating AML and relapsed leukemias, as well as the myeloproliferative and myelodysplastic disorders. Application of riskadapted therapy to patients with ALL and AML has allowed for continued improvement in long-term, event-free survival and a reduction in long-term side effects. What has been learned about leukemia biology, host pharmacogenetics, and environmental influences on leukemia development, has led to the development of effective strategies treatment and preventive strategies for childhood leukemias. There is still a significant amount of work to be done, but the future is bright.

This article can be found, with references and two additional tables, in the Reference Section on the website supporting this briefing (www.touchbriefings.com). 